\title{
Brain SPECT perfusion and PET metabolism as discordant biomarkers in major depressive disorder
}

\author{
Maud Tastevin' ', Laurent Boyer 2,3,4, Theo Korchia', Guillaume Fond 1,2,3 , Christophe Lançon 1,2, \\ Raphaëlle Richieri ${ }^{1,5}$ and Eric Guedj ${ }^{*}{ }^{*}$ (I)
}

\begin{abstract}
Background: Brain SPECT perfusion and PET metabolism have been, most often interchangeably, proposed to study the underlying pathological process in major depressive disorder (MDD). The objective of this study was to specify similarities and inconsistencies between these two biomarkers according to global characteristics of the disease. We conducted a retrospective study in 16 patients suffering from treatment-resistant MDD who underwent, during the same current episode, a cerebral perfusion SPECT with ${ }^{99 \mathrm{~m}} \mathrm{Tc}-\mathrm{HMPAO}$ and a metabolic PET with ${ }^{18} \mathrm{~F}$-FDG. Whole-brain voxel-based SPM(T) maps were generated in correlation with the number of depressive episodes and in correlation with the depression duration, separately for the two exams ( $p$-voxel $<0.001$ uncorrected, $k>20$ ).
\end{abstract}

Results: No significant correlations were found between brain metabolism and either the number of depressive episodes or the duration of the disease, even at an uncorrected p-voxel $<0.005$. On the other hand, the increased number of depressive episodes was correlated with decreased perfusion of the right middle frontal cortex, the right anterior cingulum cortex, the right insula, the right medial temporal cortex and the left precuneus. The increased depression duration was correlated with decreased perfusion of the right anterior cingulum cortex.

Conclusions: This preliminary study demonstrates more significant results with brain perfusion compared with glucose metabolism in treatment-resistant MDD, highlighting the value of brain SPECT despite less favourable instrumentation detection compared to PET.

Keywords: Treatment-resistant depression, Major depressive disorder, PET, SPECT, Biomarker

\section{Background}

Major depressive disorder (MDD) is a common mental health disorder. It is now the first cause of disability worldwide and a major contributor to the overall global burden of diseases according to the World Health Organization. It has been estimated that $15-30 \%$ of patients present with treatment-resistant depression (TRD),

\footnotetext{
*Correspondence: eric.guedj@ap-hm.fr

${ }^{6}$ Nuclear Medicine Department, APHM, CNRS, Centrale Marseille, Institut Fresnel, Timone Hospital, CERIMED, Aix Marseille University, Marseille, France

Full list of author information is available at the end of the article
}

which is resistant to antidepressants and cognitive behavioural treatment [1].

The current diagnosis and course evaluation of MDD rely on clinical examination. To date, the risk of misdiagnosis remains present because of the lack of noninvasive and quantifiable assessments of all depression dimensions [2]. Several promising treatments have been proposed (pharmacological, non-invasive and invasive neuromodulation) in the case of antidepressant resistance. Nonetheless, the pathophysiology of depression and the neural and biological mechanisms of treatment efficacy are still not fully understood. In this context, neuroimaging biomarkers are needed for diagnosing, 
predicting the course of the disorder and guiding the choice of therapy, as well as monitoring the response to these therapies.

Since 2008, with the development of the "Rdoc Research" programme, brain SPECT with ${ }^{99 \mathrm{~m}} \mathrm{Tc}$-ECD or ${ }^{99 \mathrm{~m}}$ Tc-HMPAo and ${ }^{18} \mathrm{~F}$-FDG PET have been proposed to, respectively, study regional blood flow and glucose metabolism in a range of psychiatric disorders, including MDD [3]. Despite distinct underlying mechanisms (perfusion vs. metabolism), both biomarkers are supposed to be coupled with the global synaptic activity [4]. They have been used to help in the neurological differential diagnosis of depression, and more recently considered to better understand the underlying pathological process in MDD and to predict response and non-response outcomes to neuromodulation therapies, such as repetitive transcranial magnetic stimulation (rTMS) or deep brain stimulation (DBS) [5, 6]. In this line, some brain regions seem particularly involved (e.g. the frontal cortex and more broadly the limbic system), especially in treatment-resistant cohorts. Nevertheless, the genuine overlap across PET/SPECT studies is globally more limited and slows down the clinical integration of these biomarkers into the patient's evaluation. The main explanations of these discrepancies are the small size and clinical heterogeneity of inclusions, as well as the variability of neuroimaging techniques, radiotracers and statistical models [7]. One alternative hypothesis would be that neuroimaging biomarkers are not as equivalent as anticipated in MDD, especially for perfusion and metabolism.

The objective of this study was to specify similarities and inconsistencies between brain perfusion and brain glucose metabolism according to the global characteristics of the disease (i.e. the number of previous depressive episodes and the depression duration) in a single group of patients with MDD.

\section{Methods}

Subjects

We conducted a retrospective study. The database included patients with psychiatric follow-up at Sainte Marguerite University Hospital (Marseille, France) from January 2011 through July 2019. Our inclusion criteria were patients over the age of 18 suffering from TRD who underwent a cerebral ${ }^{99 \mathrm{~m}} \mathrm{Tc}-\mathrm{HMPAo}$ SPECT and a ${ }^{18} \mathrm{~F}$ FDG PET with an interval delay of less than 18 months during a same current major depressive episode (according to DSM-IV criteria). These SPECT examinations had been performed to initially explore differential diagnoses [8]. The selected patients were subsequently included in the HrTMS trial, which included a metabolic PET imaging evaluation before treatment with ethical and regulatory authorizations (ClinicalTrials.gov: NCT02559466;
Registry Identifier ID RCB: 2015-A00345-44) [6, 9]. Patients with bipolar depression, schizophrenia or neurological comorbidities were excluded.

\section{Data collection}

The sociodemographic characteristics recorded included gender, age, marital status and education level. Clinical data included illness duration, number of depressive episodes, and melancholic and psychotic characteristics, as well as pharmaco-resistance and global severity according to the DSM-IV (SCID-IV) structured clinical interview [10]. Data concerning severity scales, such as the MADRS or Beck, were not available in all patients for the two evaluation time points. Treatment data recorded included all antidepressants, antipsychotics and mood stabilizers, as well as invasive and non-invasive brain stimulation (electroconvulsive therapy, rTMS and deep brain stimulation and vagal nerve stimulation).

\section{SPECT and PET acquisitions}

SPECT and PET scans were performed for all subjects, with the same SPECT and PET cameras, and under the same conditions. PET was performed using an integrated PET/CT camera (Discovery ST, GE Healthcare, Waukesha, WI, USA). Patients were required to fast for at least $6 \mathrm{~h}$ before undergoing the scan, with a control of normal blood glucose level. They were maintained in neurosensory resting $10 \mathrm{~min}$ before and $30 \mathrm{~min}$ after injection. ${ }^{18} \mathrm{~F}$-FDG was injected intravenously at the activity of $150 \mathrm{MBq}$, and PET images were then acquired over a period of $15 \mathrm{~min}$. Iterative reconstruction was performed on a matrix of $192 \times 192$, with correction of attenuation using CT acquisition. SPECT acquisition was performed using a double-headed rotating gamma camera (ECAM, Siemens) equipped with a fan beam collimator. Patients were maintained in neurosensory resting $10 \mathrm{~min}$ before and $20 \mathrm{~min}$ after intravenous injection of $740 \mathrm{MBq}$ of ${ }^{99 \mathrm{~m}} \mathrm{Tc}-\mathrm{HMPAO}$. The total scan time was of $25 \mathrm{~min}$ with sixty projections per head of $25 \mathrm{~s}$, collected in $128 \times 128$ format. Tomographic 3D reconstruction was performed using a filtered back-projection algorithm.

Images were initially converted from the DICOM to the NifTi format using MRIcro (www.mricro.com) and then transferred to SPM. Whole-brain statistical analysis was performed at the voxel level using SPM8 software (Wellcome Department of Cognitive Neurology, University College, London, UK) after spatial normalization (the Montreal Neurological Institute atlas) and smoothing with a Gaussian filter ( $8 \mathrm{~mm}$ full-width at half-maximum) to blur individual variations in gyral anatomy and to increase the signal-to-noise ratio. Whole-brain voxelbased $\operatorname{SPM}(\mathrm{T})$ maps were generated in correlation with the number of depressive episodes and in correlation 
with the depression duration, separately for the two exams (p-voxel $<0.001$ uncorrected, $k>20$ ), using proportional scaling with a grand mean scaled value fixed at 50 .

\section{Statistical analysis}

The Shapiro-Wilk test was used to confirm the normality of the two variables tested at PET and SPECT time examination [i.e. the depression duration $(p=0.017$ and $p=0.011$, respectively) and the number of episodes $(p<0.001)]$. Data were presented in proportions or means and standard deviations. Characteristics were compared for each patient between PET and SPECT acquisition dates using Student's t test or the Mann-Whitney $U$ test for continuous variables and the chi-square test or Fisher's exact test for categorical variables. Mean PET/ SPECT values were extracted using MARSBAR software (https://marsbar.sourceforge.net/) at the individual level for significant cluster(s) to calculate Spearman's correlations. The statistical significance level was set at $p<0.05$ in a two-sided test.

\section{Results}

Baseline characteristics

A total of 16 subjects were included in the study. Patient characteristics are described in Table 1 . Patients were mostly women with a mean age of approximately 50 years old. Our sample included 4 men and 12 women; 13 patients were single, and 10 had less than 12 years of education. They all presented with TRD with a mean number of depressive episodes estimated at $2.31 \pm 1.9$. Among this group, 13 patients suffered from a severe current episode, and melancholic criteria were present in 6 patients. The mean interval delay between PET and SPECT acquisitions was $4.37 \pm 4.89$ months ( 20 days to 13 months). No significant changes were found between these two time point evaluations, especially for clinical characteristics and treatments. Moreover, no treatment interruption occurred during the interval delay. However, age and disease duration did show statistically significant differences, with a respective difference of 5.28 months $(p=0.02)$ and 1.44 months $(p=0.023)$, respectively;

Table 1 Demographic and clinical characteristics of patients at PET and SPECT day acquisitions $(n=16)$

\begin{tabular}{|c|c|c|c|}
\hline & At PET day acquisition & At SPECT day acquisition & $p$ value \\
\hline \multicolumn{4}{|l|}{ Demographic characteristics } \\
\hline Age (years, mean $\pm S D)$ & $50.50 \pm 16.3$ & $50.06 \pm 16.7$ & 0.020 \\
\hline Education & & & 1.000 \\
\hline$>12$ years & 5 & 5 & 1.000 \\
\hline$\leq 12$ years & 10 & 10 & \\
\hline Marital status & & & 1.000 \\
\hline Single & 13 & 13 & 1.000 \\
\hline Couple & 3 & 3 & \\
\hline \multicolumn{4}{|l|}{ Clinical characteristics } \\
\hline Depression duration (months) & $136.44 \pm 120$ & $132.56 \pm 121.48$ & 0.023 \\
\hline Number of depressive episodes & $2.31 \pm 1.9$ & $2.31 \pm 1.9$ & 1.000 \\
\hline \multicolumn{4}{|l|}{ SCID severity } \\
\hline Mild & 2 & 2 & 1.000 \\
\hline Moderate & 1 & 1 & 1.000 \\
\hline Severe & 13 & 13 & 1.000 \\
\hline Melancholic features & 6 & 6 & 1.000 \\
\hline \multicolumn{4}{|l|}{ Treatments } \\
\hline SSRIS & 5 & 7 & 0.500 \\
\hline SNRIS & 6 & 5 & 0.300 \\
\hline Tricyclics & 2 & 2 & 1.000 \\
\hline MAOIs & 1 & 1 & 1.000 \\
\hline Pramipexole & 1 & 1 & 1.000 \\
\hline Antipsychotics, first generation & 1 & 1 & 1.000 \\
\hline Antipsychotics, second generation & 3 & 4 & 0.607 \\
\hline Mood stabilizers & 0 & 0 & 1.000 \\
\hline Others & 3 & 2 & 0.350 \\
\hline
\end{tabular}

Significant differences are in bold

SCID structured clinical interview for DSM-IV, SSRI selective serotonin reuptake inhibitor, SNRI serotonin-norepinephrine reuptake inhibitor, $M A O I$ monoamine oxidase inhibitors 
these limited but significant differences were expected, since SPECT was systematically performed before PET in these patients.

\section{Correlation with metabolism and perfusion}

No significant SPM(T) results were found for metabolism in correlation with either the number of depressive episodes or the depression duration, even for a less stringent threshold at an uncorrected p-voxel $<0.005$.

On the other hand, significant correlations were found with perfusion at $\mathrm{p}$-voxel $<0.001$ and $k>20$. The increased number of depressive episodes was correlated with decreased perfusion of the right middle frontal cortex (T-voxel $\max =5.50, k=106$ ), the right anterior cingulum cortex (ACC) (T-voxel $\max =5.13, k=75)$, the right medial temporal cortex (T-max $=4.93, k=79)$, the left precuneus ( $\mathrm{T}$-voxel $\max =4.80, k=66$ ) and the right insula ( $\mathrm{T}$-voxel $\max =4.52, k=61$ ). The increased depression duration was correlated with the perfusion of the right ACC (T-voxel $\max =5.31, k=58)$.
Spearman's correlations on extracted clusters confirmed a negative correlation between the number of depressive episodes and perfusion of the right medial temporal cortex $(\mathrm{rho}=-0.84, p=0.00002)$, the left precuneus (rho $=-0.50, p=0.02)$, the right $\mathrm{ACC}$ $($ rho $=-0.49, p=0.02)$ and the right insula $($ rho $=-0.58$, $p=0.01)$. Negative correlations were also confirmed between depression duration and perfusion of the right ACC (rho $=-0.61, p=0.01)$. These same clusters were extracted for brain glucose metabolism without significant correlation.

These results are presented in Figs. 1 and 2.

\section{Discussion}

We conducted a retrospective study in a sample of 16 patients suffering from TRD who all underwent a cerebral perfusion SPECT with ${ }^{99 \mathrm{~m}} \mathrm{Tc}-\mathrm{HMPAO}$ and a metabolic PET with ${ }^{18} \mathrm{~F}$-FDG. No relevant clinical changes were found upon evaluation at these two time points, especially for disease characteristics and treatments. Whole-brain voxel-based analysis revealed distinct

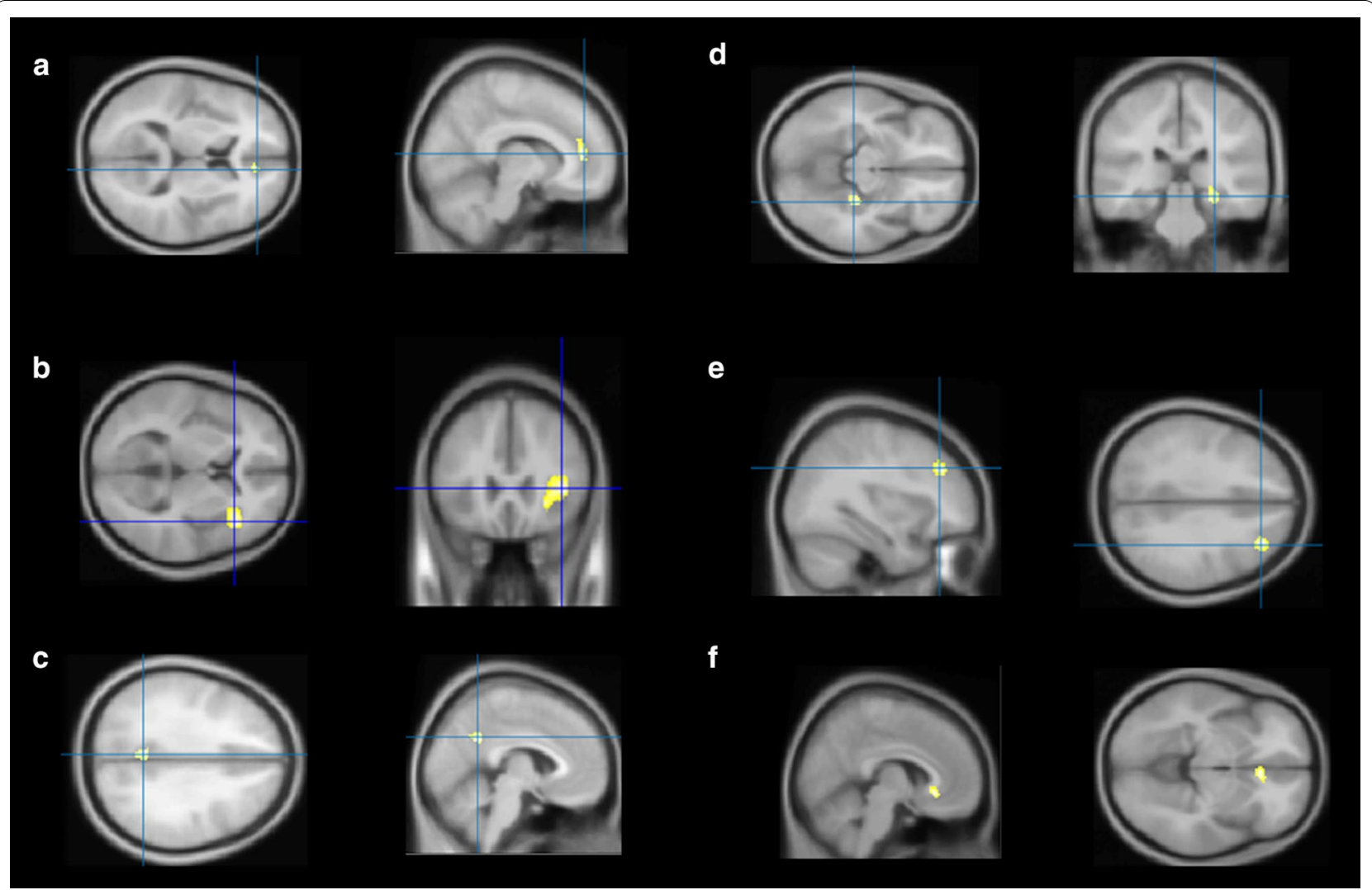

Fig. 1 Anatomical localization of significant perfusion SPECT findings ( $p$-voxel $<0.001$ uncorrected, $k>20$ ). The increased number of depressive episodes is correlated with decreased perfusion of: a the right anterior cingulate cortex (ACC) (T-voxel max $=3.85, k=75)$, $\mathbf{b}$ the right insula (T-voxel $\max =3.85, k=26)$, $\mathbf{c}$ the left precuneus (T-voxel $\max =3.85, k=62), \mathbf{d}$ the right medial temporal cortex $(\mathrm{T}$-max $=3.85, k=66)$ and $\mathbf{e}$ the right middle frontal cortex (T-voxel $\max =3.85, k=106)$. $\mathbf{f}$ The increased depression duration is correlated with decreased perfusion of the right ACC $($ T-voxel $\max =5.31, k=236)$ 

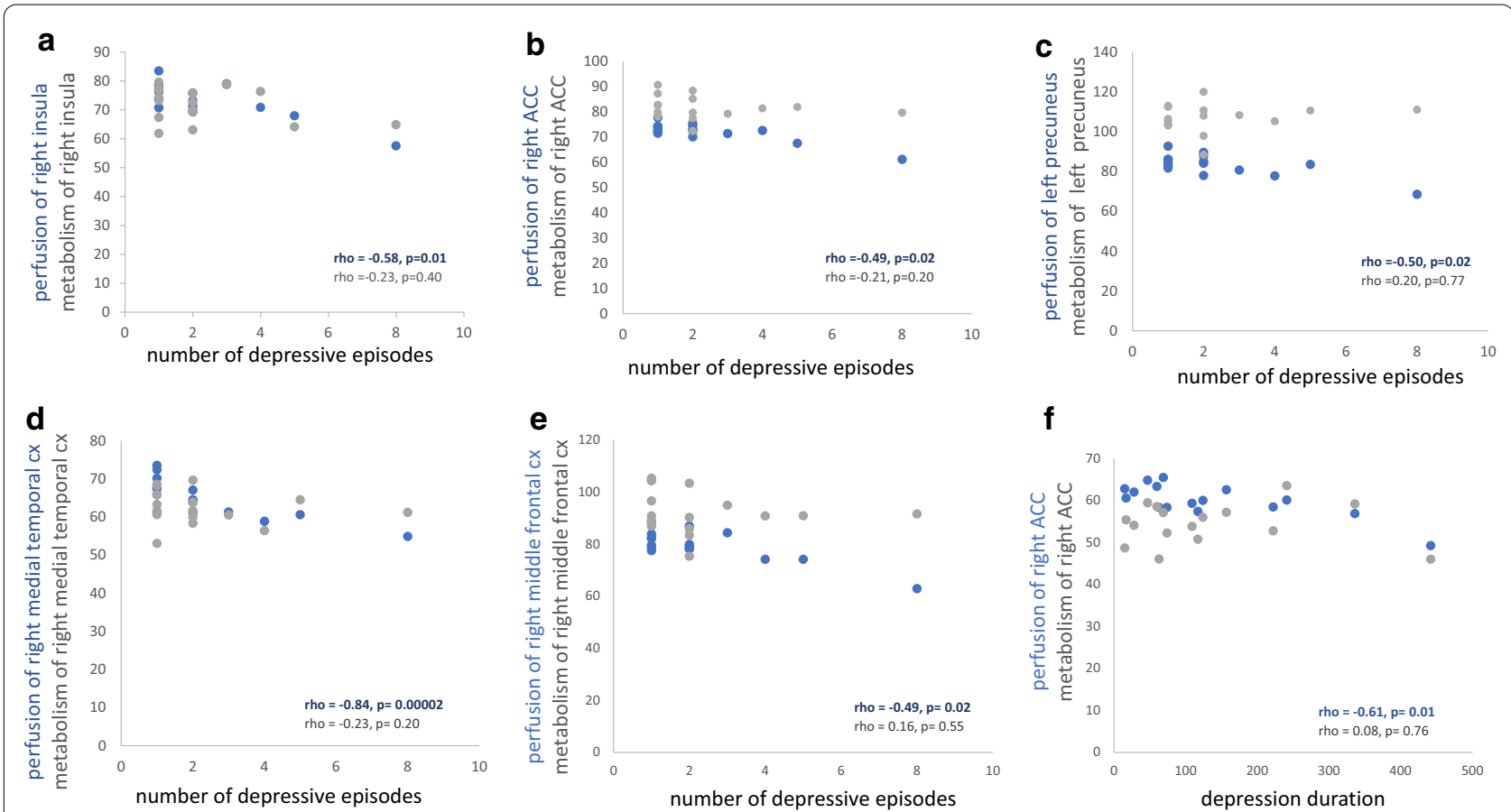

Fig. 2 Scatter plot of Spearman's correlations on extracted clusters. The increased number of depressive episodes is correlated with decreased perfusion of: a the right insula ( $r h o=-0.58, p=0.01)$, $\mathbf{b}$ the right anterior cingulate cortex (ACC) (rho $=-0.49, p=0.02$ ), $\mathbf{c}$ the left precuneus (rho $=-0.50, p=0.02)$, $\mathbf{d}$ the right medial temporal cortex (rho $=-0.84, p=0.00002)$ and $\mathbf{e}$ the right middle frontal cortex (rho $=-0.49, p=0.02)$. This correlation is not significant with the metabolism of: $\mathbf{a}$ the right insula (rho $=-0.23, p=0.40)$, $\mathbf{b}$ the right ACC ( $r h o=-0.21, p=0.20), \mathbf{c}$ the left precuneus (rho $=0.20, p=0.77$ ), $\mathbf{d}$ the right medial temporal cortex (rho $=-0.23, p=0.20$ ) and $\mathbf{e}$ the right middle frontal cortex (rho $=0.16$, $p=0.55)$. $\mathbf{f}$ The increased depression duration is correlated with decreased perfusion of the right ACC (rho $=-0.61, p=0.01)$. This correlation is not significant with the metabolism of the right $\mathrm{ACC}(\mathrm{rho}=0.08, p=0.76)$

results between perfusion and glucose metabolism; this could, at least partly, explain the previous variability of findings in the literature for these two biomarkers [7]. Significant negative correlations were found between number of episodes and perfusion of the right middle frontal cortex, the right ACC, the right insula, the right medial temporal cortex and the left precuneus, as well as between the depression duration and right ACC perfusion, while no relationship was obtained for glucose metabolism.

Number of episodes and illness duration are considered as risk factors of pharmaco-resistance and are involved in depression recurrence $[11,12]$. They also may signal risk of residual symptoms, such as sleep disturbances, executive impairments and anxiety [13]. They are considered by a group of experts as great indicators of prognosis and severity of TRD [14-16]. Identifying their relationship with cerebral functioning could improve resistance management and selection of potential cerebral therapeutic targets. Correlations with these two variables have already been described in a few previous neuroimaging studies. A voxel-based study of 127 subjects suffering from TRD showed a weak negative correlation between the duration of illness and brain ${ }^{99 \mathrm{~m}} \mathrm{Tc}-\mathrm{ECD}$ SPECT perfusion in bilateral cingulate and orbital cortices [17]. A recent MRI meta-analysis including morphometry studies found significant grey matter reduction in the rostral part of the anterior cingulum related to illness duration and repeated depressive episodes [18]. Moreover, our findings are concordant with another ${ }^{18} \mathrm{~F}$-FDG PET study. In 18 hospitalized patients with unipolar depression, Mayberg et al. [19] revealed no significant relationship between brain metabolism and illness chronicity.

The brain regions revealed in this study are known to be involved in TRD $[7,20]$. The insula is particularly involved in emotional identification and in the affective state in response to a stimulus. Resting-state hyperactivity of the insula has been linked, in MDD, to pathological self-focused mental ruminative behaviours [21]. On the other hand, the ACC modulates the link between ventral and dorsal networks involved in regulation of emotion. The dorsal ACC is specifically implicated in executive functions through the cognitive control network, and its subgenual subdivision is focused on emotional experience and processing [22]. Furthermore, the insula and ACC both belong to the salience network, 
which participates in judgement alteration and negative thoughts in MDD [23]. They also both interact with the default mode network and contribute to the alteration of attentional system and to the introspection in MDD [24]. The precuneus, medial frontal and medial temporal cortices have been mainly implicated through a default mode network and could also contribute to low selfesteem and ruminations [25]. The number of episodes and depression duration seem to alter brain perfusion, especially in cerebral areas linked to emotional and cognitive symptoms of depression. It could suggest their key role in pharmaco-resistance and recurrence by enhancing the cortico-limbic dysregulation described in unipolar depression.

PET is one of the main neuroimaging techniques evaluated in recent psychiatry research [20]. Indeed, PET is usually preferred to SPECT because of better spatial resolution. Cerebral glucose metabolism and perfusion have been considered as coupled for a long time, because the brain consumes approximately $20 \%$ of total body oxygen and $25 \%$ of total body glucose. The most important energy source for the brain is adenosine triphosphate (ATP), which is produced almost entirely by the oxidative metabolism of glucose [26]. However, their consistent correlations are presently questioned and in part justified by other mechanisms of blood flow regulation, which could produce a different cartography of cerebral perfusion from the metabolic one. This has already been highlighted in healthy subjects [27]. Furthermore, uncoupling between glucose and oxygen metabolism, via oxygen depletion and induction of downstream hypoxia response pathways, could play a key role in neurodegenerative diseases [28]. The underlying mechanisms of drug resistance in depression remain misunderstood in the current literature [29]. Nevertheless, it is not excluded that psychiatric disease could involve the same uncoupling mechanisms. Functional compensation, more specifically involving brain metabolism, may also occur. Further fundamental studies are necessary to specify the contribution of these mechanisms in depression.

The main limitations of the study were a retrospective design and a small sample size. Our results concerned patients with TRD, and they are not generalizable to all patients suffering from MDD. Subjects were mostly women with a mean age above 50 years old, which are expected sociodemographic characteristics; advanced age and female gender are risk factors for TRD [30,31]. Moreover, the interval delay between the two acquisitions was significant and could impact the findings. This difference was expected because of the sequence of exploration. Indeed, the order was fixed (PET after SPECT), with inevitably a difference using a rank test. Nevertheless, the number of depression episodes is strictly the same at the two times of the study, and the difference between depression duration was weak among the groups (136.44 \pm 120 months vs. $132.56 \pm 121.48$ months). Treatments and modifications of therapy between PET and SPECT explorations could have also impacted our findings, possibly more the metabolism than the perfusion. Nevertheless, these changes were limited and statistically non-significant. In detail, a selective serotonin reuptake inhibitor was changed for two patients, including one for a serotonin-norepinephrine reuptake inhibitor, and one antidepressant was potentialized by an antipsychotic during the interval delay, which constitute common changes in the therapeutic strategy of depression. Moreover, no treatment interruption occurred between the two examinations. To our knowledge, this is nevertheless the first study that focuses on a single group of patients to compare perfusion and metabolism in MDD. Finally, the perfusion and metabolic measurements were semiquantitative, as used in clinical practice. Further studies with absolute quantification could better explain the differences observed in this preliminary report.

\section{Conclusions}

This preliminary study demonstrates that the clinical characteristics of TRD are more significantly associated with brain perfusion than with glucose metabolism, highlighting the value of brain SPECT despite its less favourable spatial resolution and image quality compared to PET. These findings warrant other comparative studies between these two imaging techniques to provide a more frequent use of brain perfusion in future psychiatric research.

\section{Acknowledgements \\ Not applicable.}

\section{Authors' contributions}

MT analysed and interpreted the clinical and PET/SPECT data, drafted the work and gave final approval. LB analysed and interpreted the clinical and PET/ SPECT data, revised the work and gave final approval. TK acquired the clinical data, revised the work and gave final approval. GF analysed and interpreted the clinical and PET/SPECT data, revised the work and gave final approval. $\mathrm{CL}$ contributed to conception and design of the study, acquired the clinical data, revised the work and gave final approval. RR contributed to conception and design of the study, acquired, analysed and interpreted the clinical data, drafted the work and gave final approval. EG contributed to conception and design of the study, acquired, analysed and interpreted the PET/SPECT data, drafted the work and gave final approval.

\section{Funding}

This work has been carried out within DHU-Imaging with the support of the A*MIDEX Project (ANR-11-IDEX-0001-02) funded by the "Investissements d'Avenir" French Government programme managed by the French National Research Agency (ANR), the Public Assistance Marseille Hospitals (AORC junior 2014) and a research grant from a French foundation for health research and innovation "Fondation de l'Avenir".

Availability of data and materials

The data that support the findings of this study are available from the corresponding author upon reasonable request. 


\section{Ethics approval and consent to participate}

This study has been performed in accordance with the Declaration of Helsinki, with written consent of patients and the approval of the "CPP-Sud Méditerranée 1" ethics committee (ClinicalTrials.gov: NCT02559466; Registry Identifier ID RCB: 2015-A00345-44).

\section{Consent for publication}

Not applicable.

\section{Competing interests}

The authors declare that they have no competing interests.

\section{Author details}

${ }^{1}$ Department of Psychiatry, Sainte Marguerite University Hospital, Assistance Publique- Hôpitaux de Marseille, Marseille, France. ${ }^{2}$ CEReSS-Health Service Research and Quality of Life Centre, Aix Marseille University, Marseille, France. ${ }^{3}$ Department of Medical Information and Public Health, APHM, Marseille, France. ${ }^{4}$ Department of Epidemiology and Health Economics, Assistance Publique-Hôpitaux de Marseille, Marseille, France. ${ }^{5}$ CNRS, Centrale Marseille, Institut Fresnel, Aix Marseille University, Marseille, France. ${ }^{6}$ Nuclear Medicine Department, APHM, CNRS, Centrale Marseille, Institut Fresnel, Timone Hospital, CERIMED, Aix Marseille University, Marseille, France.

Received: 7 May 2020 Accepted: 24 September 2020

Published online: 08 October 2020

\section{References}

1. Thase ME. Treatment-resistant depression: prevalence, risk factors, and treatment strategies. J Clin Psychiatry. 2011;72:e18.

2. Fried El, Epskamp S, Nesse RM, Tuerlinckx F, Borsboom D. What are 'good' depression symptoms? Comparing the centrality of DSM and non-DSM symptoms of depression in a network analysis. J Affect Disord. 2016;189:314-20.

3. Zhang K, Zhu Y, Zhu Y, Wu S, Liu H, Zhang W, et al. Molecular, functional, and structural imaging of major depressive disorder. Neurosci Bull. 2016;32:273-85.

4. Magistretti PJ, Pellerin L. Cellular mechanisms of brain energy metabolism and their relevance to functional brain imaging. Philos Trans $\mathrm{R}$ Soc Lond B Biol Sci. 1999;354:1155-63.

5. Fonseka TM, MacQueen GM, Kennedy SH. Neuroimaging biomarkers as predictors of treatment outcome in major depressive disorder. J Affect Disord. 2018:233:21-35.

6. Tastevin M, Richieri R, Boyer L, Fond G, Lançon C, Guedj E. Brain PET metabolic substrate of TMS response in pharmaco-resistant depression. Brain Stimul Basic Transl Clin Res Neuromodul. 2020;13:683-5.

7. Fitzgerald PB, Laird AR, Maller J, Daskalakis ZJ. A meta-analytic study of changes in brain activation in depression. Hum Brain Mapp. 2008:29:683-95.

8. Amen DG, Krishnamani P, Meysami S, Newberg A, Raji CA. Classification of depression, cognitive disorders, and co-morbid depression and cognitive disorders with perfusion SPECT neuroimaging. J Alzheimers Dis. 2017;57:253-66.

9. Tastevin M, Baumstarck K, Groppi F, Cermolacce M, Lagrange G, Lançon C, et al. Double cone coil rTMS efficacy for treatment-resistant depression: a prospective randomized controlled trial. Brain Stimul. 2019. https://doi. org/10.1016/j.brs.2019.09.009.

10. First Michael B, Williams Janet BW, Spitzer Robert L, Gibbon M. Structured clinical interview for DSM-IV-TR Axis I disorders, Clinical Trials Version (SCID-CT); 2007.

11. Bennabi D, Aouizerate B, El-Hage W, Doumy O, Moliere F, Courtet P, et al. Risk factors for treatment resistance in unipolar depression: a systematic review. J Affect Disord. 2015:171:137-41.

12. Buckman JEJ, Underwood A, Clarke K, Saunders R, Hollon SD, Fearon P, et al. Risk factors for relapse and recurrence of depression in adults and how they operate: a four-phase systematic review and meta-synthesis. Clin Psychol Rev. 2018;64:13-38.

13. Serafini G, Nebbia J, Cipriani N, Conigliaro C, Erbuto D, Pompili M, et al. Number of illness episodes as predictor of residual symptoms in major depressive disorder. Psychiatry Res. 2018;262:469-76.
14. Bennabi D, Charpeaud T, Yrondi A, Genty J-B, Destouches S, Lancrenon $S$, et al. Clinical guidelines for the management of treatment-resistant depression: French recommendations from experts, the French Association for Biological Psychiatry and Neuropsychopharmacology and the foundation FondaMental. BMC Psychiatry. 2019;19:262. https://doi. org/10.1186/s12888-019-2237-X.

15. Gelenberg AJ, Freeman MP, Markowitz JC, Rosenbaum JF, Thase ME, Trivedi $\mathrm{MH}$ et al. American psychiatric association practice guideline for the treatment of patients with major depressive disorder, third edition. Am J Psychiatry. 2010;167:1-152.

16. Ruhé $H G$, van Rooijen G, Spijker J, Peeters FPML, Schene AH. Staging methods for treatment resistant depression. A systematic review. J Affect Disord. 2012;137:35-45.

17. Richieri R, Boyer L, Faget-Agius C, Farisse J, Mundler O, Lançon C, et al. Determinants of brain SPECT perfusion and connectivity in treatmentresistant depression. Psychiatry Res. 2015;231:134-40.

18. Bora E, Fornito A, Pantelis C, Yücel M. Gray matter abnormalities in major depressive disorder: a meta-analysis of voxel based morphometry studies. J Affect Disord. 2012;138:9-18.

19. Mayberg HS, Brannan SK, Mahurin RK, Jerabek PA, Brickman JS, Tekell JL, et al. Cingulate function in depression: a potential predictor of treatment response. NeuroReport. 1997;8:1057-61.

20. Gong B, Naveed S, Hafeez DM, Afzal Kl, Majeed S, Abele J, et al. Neuroimaging in psychiatric disorders: a bibliometric analysis of the 100 most highly cited articles. J Neuroimaging. 2019;29:14-33.

21. Sliz D, Hayley S. Major depressive disorder and alterations in insular cortical activity: a review of current functional magnetic imaging research. Front Hum Neurosci. 2012;6:323. https://doi.org/10.3389/fnhum .2012.00323.

22. Bush G, Luu P, Posner MI. Cognitive and emotional influences in anterior cingulate cortex. Trends Cogn Sci. 2000;4:215-22.

23. Manoliu A, Meng C, Brandl F, Doll A, Tahmasian M, Scherr M, et al. Insular dysfunction within the salience network is associated with severity of symptoms and aberrant inter-network connectivity in major depressive disorder. Front Hum Neurosci. 2014;7:930. https://doi.org/10.3389/fnhum .2013.00930.

24. Bessette KL, Jenkins LM, Skerrett KA, Gowins JR, DelDonno SR, Zubieta $J-K$, et al. Reliability, convergent validity and time invariance of default mode network deviations in early adult major depressive disorder. Front Psychiatry. 2018;9:244. https://doi.org/10.3389/fpsyt.2018.00244.

25. Fox MD, Snyder AZ, Vincent JL, Corbetta M, Van Essen DC, Raichle ME. The human brain is intrinsically organized into dynamic, anticorrelated functional networks. Proc Natl Acad Sci USA. 2005;102:9673-8.

26. Reilly P. Head injury: pathophysiology and management of severe closed injury. London: Chapman and Hall Medical; 1997.

27. Henriksen OM, Vestergaard MB, Lindberg U, Aachmann-Andersen NJ, Lisbjerg K, Christensen SJ, et al. Interindividual and regional relationship between cerebral blood flow and glucose metabolism in the resting brain. J Appl Physiol. 2018;125:1080-9.

28. Watts ME, Pocock R, Claudianos C. Brain energy and oxygen metabolism: emerging role in normal function and disease. Front Mol Neurosci. 2018;11:216. https://doi.org/10.3389/fnmol.2018.00216.

29. Voineskos D, Daskalakis ZJ, Blumberger DM. Management of treatmentresistant depression: challenges and strategies. Neuropsychiatr Dis Treat. 2020;16:221-34.

30. Holtzmann J, Richieri R, Saba G, Allaïli N, Bation R, Moliere F, et al. How to define treatment-resistant depression? Presse Med. 2016:45:323-8.

31. Gronemann FH, Jorgensen MB, Nordentoft M, Andersen PK, Osler M. Socio-demographic and clinical risk factors of treatment-resistant depression: a Danish population-based cohort study. J Affect Disord. 2020;261:221-9.

\section{Publisher's Note}

Springer Nature remains neutral with regard to jurisdictional claims in published maps and institutional affiliations. 